\title{
Nanoscale Mapping of Ultrafast Magnetization Dynamics with Femtosecond Lorentz Microscopy
}

\author{
Nara Rubiano da Silva, ${ }^{1}$ Marcel Möller, ${ }^{1}$ Armin Feist, ${ }^{1}$ Henning Ulrichs, ${ }^{2}$ Claus Ropers, ${ }^{1}$ and Sascha Schäfer ${ }^{1, *}$ \\ ${ }^{1}$ University of Göttingen, IV. Physical Institute, Göttingen 37077, Germany \\ ${ }^{2}$ University of Göttingen, I. Physical Institute, Göttingen 37077, Germany
}

(Received 28 November 2017; revised manuscript received 28 February 2018; published 29 August 2018)

\begin{abstract}
Novel time-resolved imaging techniques for the investigation of ultrafast nanoscale magnetization dynamics are indispensable for further developments in light-controlled magnetism. Here, we introduce femtosecond Lorentz microscopy, achieving a spatial resolution below $100 \mathrm{~nm}$ and a temporal resolution of $700 \mathrm{fs}$, which gives access to the transiently excited state of the spin system on femtosecond timescales and its subsequent relaxation dynamics. We demonstrate the capabilities of this technique by spatiotemporally mapping the light-induced demagnetization of a single magnetic vortex structure and quantitatively extracting the evolution of the magnetization field after optical excitation. Tunable electron imaging conditions allow for an optimization of spatial resolution or field sensitivity, enabling future investigations of ultrafast internal dynamics of magnetic topological defects on a $10 \mathrm{~nm}$ length scale.
\end{abstract}

DOI: 10.1103/PhysRevX.8.031052

Subject Areas: Magnetism, Nanophysics, Photonics

Being a key aspect for future processing and storage applications, strategies for the manipulation of nanoscale magnetic domains and topological defects were recently developed utilizing various stimuli such as electrical current [1-5] and light [6-11]. Optical control of magnetic nanostructures is particularly appealing due to the absence of an applied external field and the possibility for ultrafast switching speeds. The underlying physical mechanisms, such as direct spin-light interactions, optically driven spin currents, and spin-flip scattering and the collapse of exchange splitting in a hot electron environment, are active fields of study and their relative importance in different material systems is still debated [12-16]. The relevant processes occur on femtosecond timescales, before the spin, electron, and lattice subsystems are thermalized. For further progress, it is essential to develop the experimental tools to access the optically induced magnetization reordering processes at their intrinsic nanometer spatial and femtosecond temporal scales. In particular, imaging methods can play a decisive role for the full understanding of such processes. However, it is challenging to achieve a combined femtosecond temporal and nanometer spatial resolution within the same magnetic imaging method, despite recent advances in the fields of ultrafast optical microscopy using ultrashort visible [17] and x-ray pulses

*sascha.schaefer@phys.uni-goettingen.de

Published by the American Physical Society under the terms of the Creative Commons Attribution 4.0 International license. Further distribution of this work must maintain attribution to the author(s) and the published article's title, journal citation, and DOI.
[3,18-21], including lab-scale approaches with circularly polarized high-harmonic radiation [22].

Electron-based magnetic imaging techniques, such as electron holography [23,24], Lorentz microscopy [4,25,26], and spin-polarization analysis of secondary electrons [27], can routinely reach spatial resolutions down to a few nanometers. By utilizing fast detectors, dynamic magnetic processes were captured in the millisecond to nanosecond range [28-34]. To overcome the limitation on detector response times and further improve the achievable temporal resolution, pulsed electron sources are employed in stroboscopic schemes. In particular, stroboscopic Lorentz microscopy was used for the investigation of field-assisted or laser-excited domain wall movement and magnetic phase transitions [35-37].

Whereas laser-driven photocathodes are available that deliver electron pulses down to the femtosecond range [38-41], previous time-resolved Lorentz microscopy experiments were restricted to the picosecond [37] and nanosecond timescales $[36,42]$, due to the limited coherence properties of these electron sources. In recent years, high-brightness nanoscale photocathode geometries were developed [43-45], which now offer a new path to push Lorentz microscopy into the femtosecond temporal regime and allow us to address optically excited transient spin systems before local thermalization.

Here, we introduce real-space nanoscale mapping of light-induced magnetization dynamics by ultrafast transmission electron microscopy (UTEM) with femtosecond temporal resolution. We quantitatively track the timedependent magnetization field in a single vortex structure during laser-driven ultrafast demagnetization, reaching simultaneously 700-fs temporal and below-100-nm spatial 
resolution. These results demonstrate the capability of femtosecond Lorentz microscopy for the imaging of transient magnetization fields on timescales faster than the spin-lattice equilibration.

In our experiments, we study an isolated magnetic permalloy disc (1- $\mu \mathrm{m}$ diameter, 20 -nm thickness), prepared by electron-beam lithography on a silicon nitride membrane (50-nm thickness). The ground state texture of the disc consists of a magnetic vortex state, for which an inplane oriented magnetization field $\vec{M}(\vec{r})$ of constant magnitude $|\vec{M}(\vec{r})|=M_{s}$ curls around the center of the structure [46-48]. In the vortex core, with a typical diameter on the 10-nm scale [49], the magnetization turns to an out-ofplane direction.

Transmission electron microscopy under out-of-focus imaging conditions, so-called Fresnel mode Lorentz microscopy [50], provides for an image contrast which is sensitive to the in-plane magnetization field [Fig. 1(a)]. For the vortex sample, a conically shaped Aharonov-Bohm phase shift $\phi$ [51] is imprinted onto an incident electron wavefront,

$$
\phi=\frac{e}{\hbar}\left(\frac{1}{v} \int V(\vec{r}, z) d s-\int \vec{A}(\vec{r}, z) \cdot \mathrm{d} \vec{s}\right),
$$

where $V$ and $\vec{A}$ are the electrostatic and magnetic vector potentials, respectively; $e$ the electron charge; $\hbar$ the reduced Planck constant; $v$ the speed of the imaging electron; and the integral is computed along the electron beam trajectory. In defocused electron images, this spatial phase information is transferred to changes in electron image intensity, which, in general, can be employed to reconstruct the magnetic induction by using phase retrieval approaches such as the transport-of-intensity equation $[7,52,53]$. For the magnetic vortex state considered here [Fig. 1(b), upper left panel], the most prominent feature in the defocused electron micrograph is a bright or dark spot in the center of the magnetic disc. This maximum or minimum in electron intensity is caused by the lensing effect of the conical phase shift, which depends on the curling direction of the magnetic vortex and the sign of defocus. Furthermore, for a given sample thickness, known electrostatic potential, and fixed defocus value, the saturation magnetization $M_{s}$ can be extracted from the image intensity in the disc center (see below) [54].

In order to perform time-resolved stroboscopic Lorentz microscopy, we employed a recently developed pulsed electron source based on nanolocalized photoemission from a Schottky field emitter with a tunable pulse duration reaching $200 \mathrm{fs}[45,55]$. The large electrostatic extraction field at the emitter minimizes space-charge induced temporal broadening of the electron pulses. In addition, the small source size of the emitter yields electron pulses with a large transverse coherence, which we previously harnessed as a nanometer-focused probe for scanning and local (a)
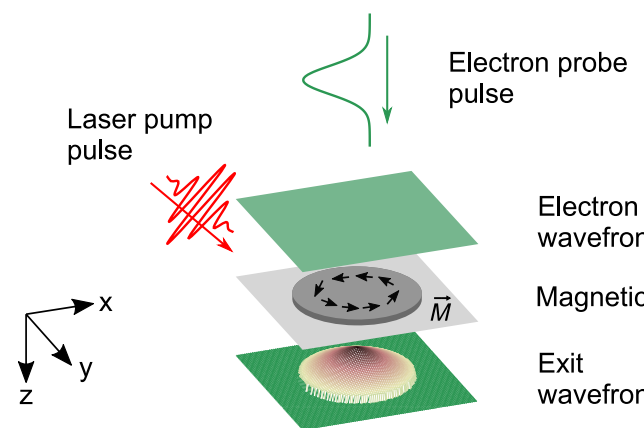

Electron wavefront

Magnetic disc

Exit wavefront

Microscope imaging system
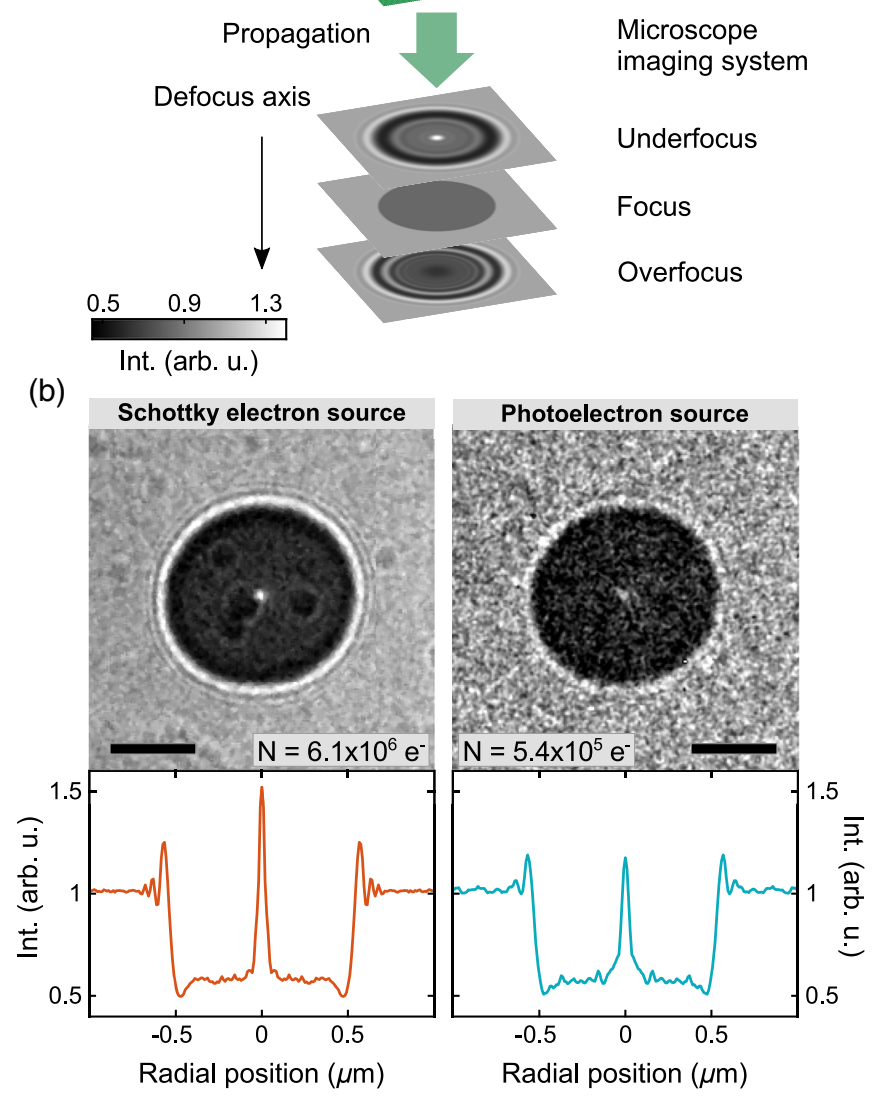

FIG. 1. Femtosecond Lorentz microscopy. (a) Scheme of experimental setup and Lorentz image formation. (b) Lorentz micrographs of the permalloy disc using a continuous Schottky emission (left) and laser-triggered photoemission (right) recorded with the same microscope conditions $(-1.5-\mathrm{mm}$ defocus). The number of electrons contributing to each image is indicated. Total illuminated area is about $25 \mu \mathrm{m}$ in diameter. Profiles in the lower panels show the azimuthally averaged intensity of the corresponding micrographs. Scale bars represent $500 \mathrm{~nm}$.

probing techniques $[55,56]$. We now make use of the large spatial coherence by spreading the electron beam for phasecontrast imaging. The present experiments are performed at a $500-\mathrm{kHz}$ repetition rate, and employ pulses with a duration of $700 \mathrm{fs}$, containing up to 1 electron/pulse at the sample. Figure 1(b) (right panels) displays a Lorentz micrograph of the vortex structure collected using $1.5 \times 10^{8}$ photoelectron pulses. Besides the resolution, the visibility of the bright spot at the center and of Fresnel fringes at the 
edge of the disc is largely retained, albeit with a lower number of imaging electrons, demonstrating comparable spatial coherence of the photoelectron source to a thermal Schottky emitter [Fig. 1(b), left panels]. For the pulsed photoelectron illumination in the time-resolved experiments, a transverse coherence length of $76 \mathrm{~nm}$ was utilized [54]. Because of the sparse electron distribution for imaging, long exposure times are required in our experiments. To ensure that drift is not a limiting factor for the spatial resolution, we used a drift-correction scheme based on the disc and marker bars on the substrate to align multiple short-exposure micrographs [54].

We optically excite the magnetic vortex structure with femtosecond laser pulses [50-fs duration, 800-nm center wavelength, about $50-\mu \mathrm{m}$ full-width-at-half-maximum (FWHM) spot diameter, p-polarized at a $55^{\circ}$ angle of incidence], and probe the transient magnetic structure with ultrashort electron pulses as a function of delay time $\Delta t$ between pump and probe pulses. Figure 2(a) shows a set of Lorentz micrographs (6 minutes exposure time each, accumulating $1.8 \times 10^{8}$ electron pulses, -4.5 -mm defocus) for varying delay times and two optical excitation fluences. Directly after excitation $(\Delta t=0.1 \mathrm{ps})$, we observe a strong decrease in the intensity of the bright central spot, followed by a partial recovery on a picosecond timescale. Selected image intensity profiles (azimuthally averaged around the vortex core) are shown in Fig. 2(c).

Considering a larger number of delay times, Fig. 2(b) displays the time evolution of the averaged radial intensity profiles of the Lorentz micrographs (upper panel) and the corresponding image intensity changes (lower panel). Adapting Lorentzian line profiles with amplitude $A$ to the intensity near the disc center, we extract the delaydependent normalized amplitude $A(\Delta t) / A_{0}\left(A_{0}\right.$ is the mean amplitude without optical excitation). For an analysis of the delay-dependent traces [Fig. 2(d)], we adopt an exponential recovery model [Fig. 2(d), solid lines] given by

$$
A(\Delta t)=A(\Delta t<0)-H(\Delta t)\left[a-b\left(1-\mathrm{e}^{-\Delta t / t_{\mathrm{rec}}}\right)\right],
$$

where $H(\Delta t)$ is the Heaviside step function, $A(\Delta t<0)$ the amplitude for negative delay times, $a$ the amplitude of the initial drop, and $b$ the amplitude of the partial contrast recovery at later delay times. The finite electron pulse duration is included by convolution of the expression in Eq. (2) with a corresponding Gaussian envelope. The time

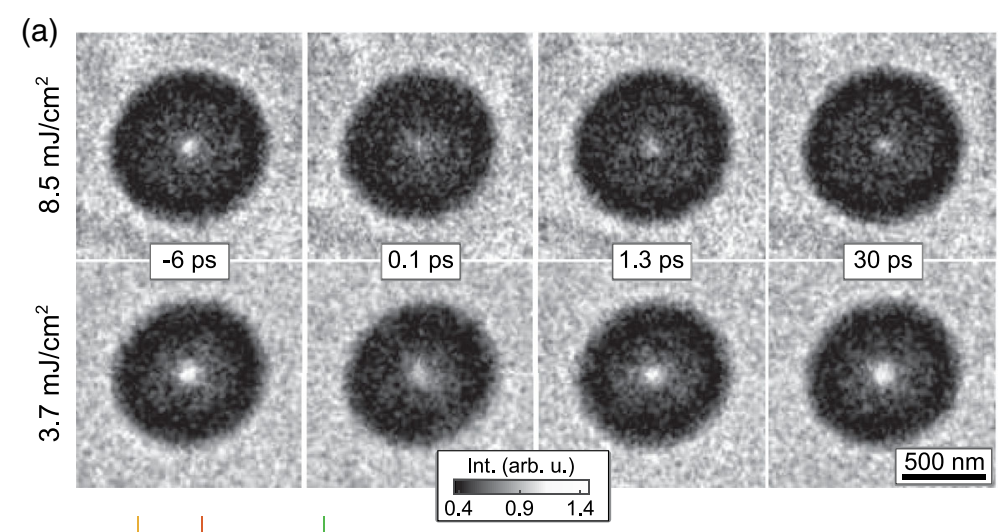

(b)

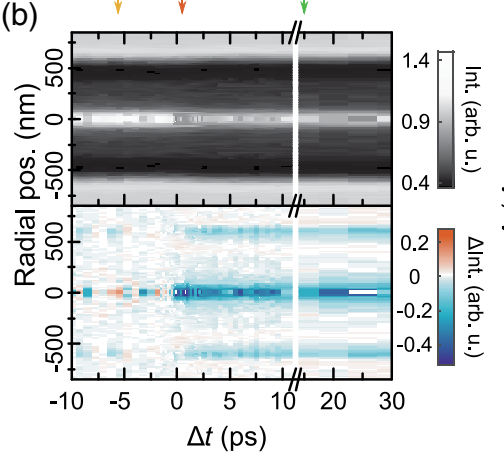

(d)

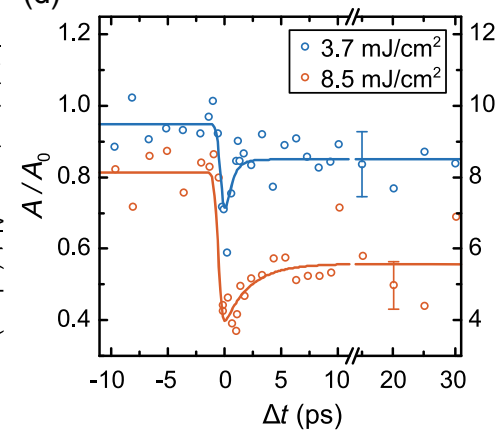

(c)

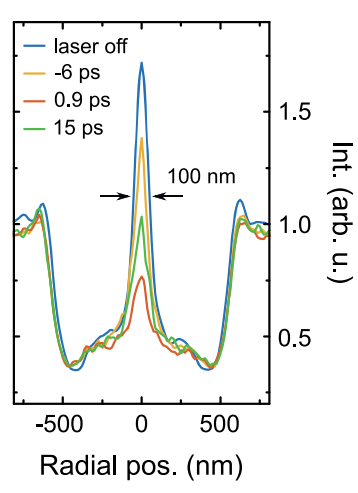

(e)

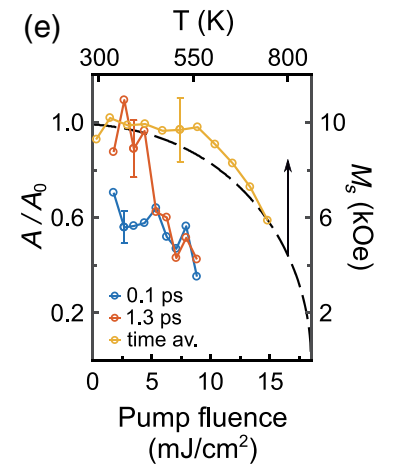

FIG. 2. Delay-time-dependent Lorentz contrast of a single, optically excited magnetic disc. (a) Time-resolved Lorentz micrographs for pump fluences of 8.5 (top row) and $3.7 \mathrm{~mJ} / \mathrm{cm}^{2}$ (bottom row). (b) Temporal evolution of azimuthally averaged intensity profiles of the micrographs for a pump fluence of $8.5 \mathrm{~mJ} / \mathrm{cm}^{2}$ (top row). In the bottom row, the mean profile before the arrival of the pump pulse is subtracted. Profiles for the marked delay times are shown in (c), along with a profile of the unpumped disc. (d) Temporal evolution of the image amplitude $A$ at the disc center (normalized by the amplitude $A_{0}$ for the unpumped structure). Solid lines represent the exponential recovery model. (e) The amplitude $A$ as a function of pump fluence from micrographs obtained using a continuous electron beam and electron pulses at specific time delays. The black dashed curve represents Weiss molecular field theory adapted to the data. 
traces are well described by recovery time constants of about $t_{\text {rec }}=0.6$ and $2.2 \mathrm{ps}$ for the low and high fluence case, respectively.

The observed contrast evolution can be attributed to the laser-induced spin heating and subsequent demagnetization of the disc. In a simple three-temperature-model (3TM) $[14,57]$, which considers individually thermalized electron, spin, and lattice subsystems, optical excitation results in an ultrafast drop of magnetization due to rapid electron-spin coupling on a timescale of a few $100 \mathrm{fs}$. The subsequent coupling with the lattice bath leads to a partial recovery of the sample magnetization. The corresponding timescale $t_{\text {rec }}$ on the order of $1 \mathrm{ps}$ is resolved in our experiments and is in agreement with previous ultrafast optical spectroscopy experiments $[58,59]$. In addition to the pulsed heating of the spin system, the optical power absorbed by the sample results in an increased average temperature and, thereby, reduced magnetization. For increasing fluence, this effect can be observed in the experimental data as a contrast reduction at negative time delays.

A unique feature of ultrafast Lorentz microscopy is the direct connection of image intensity changes to the transient magnetization distribution. For a quantitative analysis, we perform electron image simulations [54] for a vortex state with varying saturation magnetization $M_{s}$ [with fixed $\vec{M}(\vec{r}) / M_{s}$ ] [60]. The dependence of the radial image profiles on the saturation magnetization $M_{s}$ [Fig. 3(c)] demonstrates that, for the chosen imaging conditions, the amplitude $A$ of the central image spot is approximately proportional to $M_{s}$.

Using a linear relation between $A$ and $M_{s}$, we can now extract the delay-dependent magnetization $M_{s}(\Delta t)$ from the fluence-dependent transient amplitude changes in Figs. 2(d) and 2(e). The time-averaged saturation magnetization [Fig. 2(e), yellow curve] follows a behavior which can be described within the Weiss molecular field theory [61] (broken black curve) and originates from the average sample heating under laser illumination. For the present sample system, average sample heating is reduced by placing a single isolated permalloy disc on an optically transparent silicon nitride substrate. From the time-resolved Lorentz micrographs at $\Delta t=0.1 \mathrm{ps}$, we extract a drop in saturation magnetization to about 3.7-7.0 $\pm 0.7 \mathrm{kOe}$ in the considered fluence range. For example, for a fluence of $8.5 \mathrm{~mJ} / \mathrm{cm}^{2}$ [Fig. 2(d), red curve], a minimum magnetization of $3.7 \pm 0.7 \mathrm{kOe}$ is found for the hot spin system at $\Delta t=0.9 \mathrm{ps}$, recovering to $5.6 \pm 0.7 \mathrm{kOe}$ at longer delay times (corresponding temperature of about $753 \mathrm{~K}$ ) [60].

These results demonstrate the capabilities of ultrafast Lorentz microscopy for the nanoscale mapping and quantitative characterization of magnetization dynamics and suggest its application for high-resolution real-space investigations of ultrafast magnetic phenomena. To further gauge the achievable spatial resolution, we consider simulated Lorentz images of the vortex at different defoci and compare them to experimental Lorentz micrographs. The extracted Lorentz profiles, shown in Fig. 3(a), are in convincing agreement with the experimental data. The vortex in the present geometry is estimated to have a core diameter of about $26 \mathrm{~nm}$ [49]. At the $-4.5-\mathrm{mm}$ defocus adopted in the time-resolved experiments, the central image spot is broadened to about $100 \mathrm{~nm}$ (FWHM), and the detailed structure of the magnetization field close to the core is largely unresolved. The resolution is considerably enhanced for smaller defoci, but with reduced image contrast. Figure 3(b) shows the resolution and contrast of the vortex core region when imaged with electron pulses

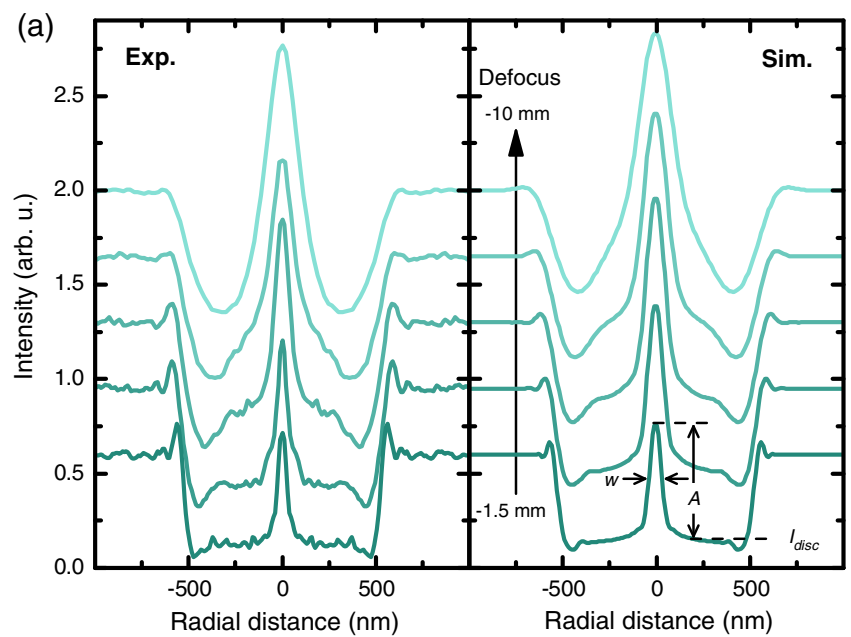

(b)

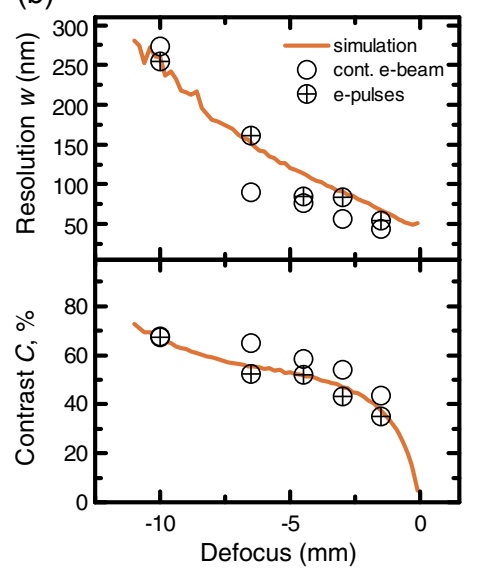

(c)

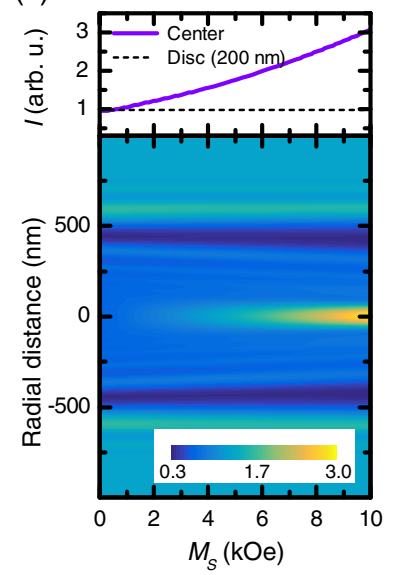

FIG. 3. Spatial resolution and quantitative image analysis in femtosecond Lorentz microscopy. (a) Radial profiles of experimental micrographs (left panel) and simulated images (right panel) at defoci of $-10,-6.5,-4.5,-3.0$, and $-1.5 \mathrm{~mm}$. Micrographs were obtained using the same illumination conditions and electron pulse durations as for the time-resolved experiments. (b) Resolution (upper panel, full width at half maximum $w$ of the central peak) and contrast $C$ [lower panel, $C=A /\left(2 I_{\text {disc }}\right), I_{\text {disc }}$ at $200 \mathrm{~nm}$ from the center] extracted from the radial profiles for different defocus values. (c) Radial profiles of simulated Lorentz images (lower panel) demonstrate an approximate proportionality between the image intensity at the disc center and saturation magnetization (upper panel). 
(circle plus symbols) and with Schottky-emitted electrons (open circles). The comparable resolution in both cases demonstrates that transient magnetic structures can be imaged with similar quality as with conventional Lorentz microscopy.

Notably, at small defoci, a higher electron dose is required to resolve changes in the low contrast images. From the contrast in the simulated images, we estimate the minimum electron dose required for resolving the bright spot of area $\pi w^{2} / 4$ just above the shot noise level as $1 / C^{2}$, which, e.g., yields about 600 electrons $/ \mu \mathrm{m}^{2}$ for $-4.5-\mathrm{mm}$ defocus and 20000 electrons $/ \mu \mathrm{m}^{2}$ for a defocus of $-500 \mu \mathrm{m}$. Experimentally, for the current electron pulse parameters and at a defocus of $-1.5 \mathrm{~mm}$, we could achieve a resolution of $55 \pm 2 \mathrm{~nm}$ within a reasonable exposure time of $5 \mathrm{~min}$. A further increase in the spatial resolution of ultrafast Lorentz microscopy is expected, if high-end electron detectors are employed, which push the signal-tonoise limit in the images close to the shot-noise limit. In addition, temporal resolution may be improved by employing compression schemes to reduce electron pulse duration, such as the ones based on radio-frequency cavities [62-64], terahertz $[65,66]$ and optical radiation $[67,68]$.

With a femtosecond temporal resolution now available in Lorentz microscopy, ultrafast magnetic processes are accessible, in which the exchange and spin-orbit interaction play a dominant role. One example is the real-space imaging of light-induced superdiffusive spin currents in nanoscale geometries. In addition, femtosecond Lorentz microscopy may open a route to probe the interaction of spins with intense light fields, as applied in all-optical magnetic switching schemes.

In conclusion, we demonstrated the mapping of ultrafast demagnetization dynamics in a single nanostructure with sub-100-nm-spatial and 700-fs-temporal resolution by femtosecond Lorentz microscopy. Femtosecond Lorentz microscopy complements existing x-ray-based techniques and contributes a unique laboratory-based access to nanometer-femtosecond magnetization processes. In the future, this approach may enable the real-space imaging of nanoscale spin systems interacting with intense light fields with a broad range of applications, including the investigation of spintronic terahertz emitters or all-optical switching schemes.

We gratefully acknowledge funding by the Deutsche Forschungsgemeinschaft (DFG-SPP-1840 "Quantum Dynamics in Tailored Intense Fields," and DFG-SFB1073 "Atomic Scale Control of Energy Conversion," projects A05 and A06), support by the Lower Saxony Ministry of Science and Culture and funding of the instrumentation by the DFG and VolkswagenStiftung. N.R.S. would like to acknowledge the support by the Conselho Nacional de Desenvolvimento Científico e Tecnológico (Science Without Borders Program, Governo Dilma Rousseff, Brazil).
[1] A. Ruotolo, V. Cros, B. Georges, A. Dussaux, J. Grollier, C. Deranlot, R. Guillemet, K. Bouzehouane, S. Fusil, and A. Fert, Phase-Locking of Magnetic Vortices Mediated by Antivortices, Nat. Nanotechnol. 4, 528 (2009).

[2] W. Jiang, P. Upadhyaya, W. Zhang, G. Yu, M. B. Jungfleisch, F. Y. Fradin, J. E. Pearson, Y. Tserkovnyak, K. L. Wang, O. Heinonen, S. G. E. te Velthuis, and A. Hoffmann, Blowing Magnetic Skyrmion Bubbles, Science 349, 283 (2015).

[3] B. Van Waeyenberge, A. Puzic, H. Stoll, K. W. Chou, T. Tyliszczak, R. Hertel, M. Fähnle, H. Brückl, K. Rott, G. Reiss, I. Neudecker, D. Weiss, C. H. Back, and G. Schütz, Magnetic Vortex Core Reversal by Excitation with Short Bursts of an Alternating Field, Nature (London) 444, 461 (2006).

[4] S. D. Pollard, L. Huang, K. S. Buchanan, D. A. Arena, and Y. Zhu, Direct Dynamic Imaging of Non-adiabatic Spin Torque Effects, Nat. Commun. 3, 1028 (2012).

[5] J. Torrejon, M. Riou, F. A. Araujo, S. Tsunegi, G. Khalsa, D. Querlioz, P. Bortolotti, V. Cros, K. Yakushiji, A. Fukushima, H. Kubota, S. Yuasa, M. D. Stiles, and J. Grollier, Neuromorphic Computing with Nanoscale Spintronic Oscillators, Nature (London) 547, 428 (2017).

[6] M. Finazzi, M. Savoini, A. R. Khorsand, A. Tsukamoto, A. Itoh, L. Duò, A. Kirilyuk, Th. Rasing, and M. Ezawa, LaserInduced Magnetic Nanostructures with Tunable Topological Properties, Phys. Rev. Lett. 110, 177205 (2013).

[7] T. Eggebrecht, M. Möller, J. G. Gatzmann, N. R. da Silva, A. Feist, U. Martens, H. Ulrichs, M. Münzenberg, C. Ropers, and S. Schäfer, Light-Induced Metastable Magnetic Texture Uncovered by In Situ Lorentz Microscopy, Phys. Rev. Lett. 118, 097203 (2017).

[8] X. Fu, S. D. Pollard, B. Chen, B.-K. Yoo, H. Yang, and Y. Zhu, Optical Manipulation of Magnetic Vortices Visualized In Situ by Lorentz Electron Microscopy, Sci. Adv. 4, eaat3077 (2018).

[9] C. von Korff Schmising, B. Pfau, M. Schneider, C. M. Günther, M. Giovannella, J. Perron, B. Vodungbo, L. Müller, F. Capotondi, E. Pedersoli, N. Mahne, J. Lüning, and S. Eisebitt, Imaging Ultrafast Demagnetization Dynamics after a Spatially Localized Optical Excitation, Phys. Rev. Lett. 112, 217203 (2014).

[10] L. Le Guyader, S. El Moussaoui, M. Buzzi, R. V. Chopdekar, L. J. Heyderman, A. Tsukamoto, A. Itoh, A. Kirilyuk, Th. Rasing, A. V. Kimel, and F. Nolting, Demonstration of Laser Induced Magnetization Reversal in GdFeCo Nanostructures, Appl. Phys. Lett. 101, 022410 (2012).

[11] T.-M. Liu, T. Wang, A. H. Reid, M. Savoini, X. Wu, B. Koene, P. Granitzka, C. E. Graves, D. J. Higley, Z. Chen, G. Razinskas, M. Hantschmann, A. Scherz, J. Stöhr, A. Tsukamoto, B. Hecht, A. V. Kimel, A. Kirilyuk, Th. Rasing, and H. A. Dürr, Nanoscale Confinement of All-Optical Magnetic Switching in TbFeCo-Competition with Nanoscale Heterogeneity, Nano Lett. 15, 6862 (2015).

[12] A. V. Kimel, A. Kirilyuk, P. A. Usachev, R. V. Pisarev, A. M. Balbashov, and Th. Rasing, Ultrafast Non-thermal Control of Magnetization by Instantaneous Photomagnetic Pulses, Nature (London) 435, 655 (2005).

[13] F. Hansteen, A. Kimel, A. Kirilyuk, and Th. Rasing, Femtosecond Photomagnetic Switching of Spins in Ferrimagnetic Garnet Films, Phys. Rev. Lett. 95, 047402 (2005). 
[14] B. Koopmans, G. Malinowski, F. Dalla Longa, D. Steiauf, M. Fähnle, T. Roth, M. Cinchetti, and M. Aeschlimann, Explaining the Paradoxical Diversity of Ultrafast LaserInduced Demagnetization, Nat. Mater. 9, 259 (2010).

[15] M. Battiato, K. Carva, and P. M. Oppeneer, Superdiffusive Spin Transport as a Mechanism of Ultrafast Demagnetization, Phys. Rev. Lett. 105, 027203 (2010).

[16] R. John, M. Berritta, D. Hinzke, C. Müller, T. Santos, H. Ulrichs, P. Nieves, J. Walowski, R. Mondal, O. ChubykaloFesenko, J. McCord, P. M. Oppeneer, U. Nowak, and M. Münzenberg, Magnetisation Switching of FePt Nanoparticle Recording Medium by Femtosecond Laser Pulses, Sci. Rep. 7, 4114 (2017).

[17] B. Mozooni, T. von Hofe, and J. McCord, Picosecond WideField Magneto-optical Imaging of Magnetization Dynamics of Amorphous Film Elements, Phys. Rev. B 90, 054410 (2014).

[18] S.-B. Choe, Y. Acremann, A. Scholl, A. Bauer, A. Doran, J. Stöhr, and H. A. Padmore, Vortex Core-Driven Magnetization Dynamics, Science 304, 420 (2004).

[19] A. Bisig, C. A. Akosa, J.-H. Moon, J. Rhensius, C. Moutafis, A. von Bieren, J. Heidler, G. Kiliani, M. Kammerer, M. Curcic, M. Weigand, T. Tyliszczak, B. Van Waeyenberge, H. Stoll, G. Schütz, K.-J. Lee, A. Manchon, and M. Kläui, Enhanced Nonadiabaticity in Vortex Cores due to the Emergent Hall Effect, Phys. Rev. Lett. 117, 277203 (2016).

[20] B. Pfau et al., Ultrafast Optical Demagnetization Manipulates Nanoscale Spin Structure in Domain Walls, Nat. Commun. 3, 1100 (2012).

[21] Felix Büttner, C. Moutafis, M. Schneider, B. Krüger, C. M. Günther, J. Geilhufe, C. v Korff Schmising, J. Mohanty, B. Pfau, S. Schaffert, A. Bisig, M. Foerster, T. Schulz, C. a. F. Vaz, J. H. Franken, H. J. M. Swagten, M. Kläui, and S. Eisebitt, Dynamics and Inertia of Skyrmionic Spin Structures, Nat. Phys. 11, 225 (2015).

[22] O. Kfir, S. Zayko, C. Nolte, M. Sivis, M. Möller, B. Hebler, S. S. P. K. Arekapudi, D. Steil, S. Schäfer, M. Albrecht, O. Cohen, S. Mathias, and C. Ropers, Nanoscale Magnetic Imaging using Circularly Polarized High-Harmonic Radiation, Sci. Adv. 3, eaao4641 (2017).

[23] A. Tonomura, Applications of Electron Holography, Rev. Mod. Phys. 59, 639 (1987).

[24] C. Jin, Z.-A. Li, A. Kovács, J. Caron, F. Zheng, F. N. Rybakov, N. S. Kiselev, H. Du, S. Blügel, M. Tian, Y. Zhang, M. Farle, and R. E. Dunin-Borkowski, Control of Morphology and Formation of Highly Geometrically Confined Magnetic Skyrmions, Nat. Commun. 8, 15569 (2017).

[25] J. N. Chapman, The Investigation of Magnetic Domain Structures in Thin Foils by Electron Microscopy, J. Phys. D 17, 623 (1984).

[26] X. Z. Yu, Y. Onose, N. Kanazawa, J. H. Park, J. H. Han, Y. Matsui, N. Nagaosa, and Y. Tokura, Real-Space Observation of a Two-Dimensional Skyrmion Crystal, Nature (London) 465, 901 (2010).

[27] K. Koike, Spin-Polarized Scanning Electron Microscopy, Microscopy 62, 177 (2013).

[28] T. Matsuda, A. Fukuhara, T. Yoshida, S. Hasegawa, A. Tonomura, and $\mathrm{Q}$. $\mathrm{Ru}$, Computer Reconstruction from
Electron Holograms and Observation of Fluxon Dynamics, Phys. Rev. Lett. 66, 457 (1991).

[29] T. Matsuda, K. Harada, H. Kasai, O. Kamimura, and A. Tonomura, Observation of Dynamic Interaction of Vortices with Pinning Centers by Lorentz Microscopy, Science 271, 1393 (1996).

[30] I. S. Weir, J. N. Chapman, I. S. Molchanov, D. M. Titterington, and J. Rose, Observation and Modelling of Magnetization Reversal in Multilayers Supporting Perpendicular Magnetization, J. Phys. D 32, 395 (1999).

[31] S. Pöllath, J. Wild, L. Heinen, T. N. G. Meier, M. Kronseder, L. Tutsch, A. Bauer, H. Berger, C. Pfleiderer, J. Zweck, A. Rosch, and C.H. Back, Dynamical Defects in Rotating Magnetic Skyrmion Lattices, Phys. Rev. Lett. 118, 207205 (2017).

[32] M. Mochizuki, X.Z. Yu, S. Seki, N. Kanazawa, W. Koshibae, J. Zang, M. Mostovoy, Y. Tokura, and N. Nagaosa, Thermally Driven Ratchet Motion of a Skyrmion Microcrystal and Topological Magnon Hall Effect, Nat. Mater. 13, 241 (2014).

[33] J. Rajeswari, P. Huang, G. F. Mancini, Y. Murooka, T. Latychevskaia, D. McGrouther, M. Cantoni, E. Baldini, J. S. White, A. Magrez, T. Giamarchi, H. M. Rønnow, and F. Carbone, Filming the Formation and Fluctuation of Skyrmion Domains by Cryo-Lorentz Transmission Electron Microscopy, Proc. Natl. Acad. Sci. U.S.A. 112, 14212 (2015).

[34] R. Frömter, F. Kloodt, S. Rößler, A. Frauen, P. Staeck, D. R. Cavicchia, L. Bocklage, V. Röbisch, E. Quandt, and H. P. Oepen, Time-Resolved Scanning Electron Microscopy with Polarization Analysis, Appl. Phys. Lett. 108, 142401 (2016).

[35] O. Bostanjoglo and Th. Rosin, Resonance Oscillations of Magnetic Domain Walls and Bloch Lines Observed by Stroboscopic Electron Microscopy, Phys. Status Solidi A 57, 561 (1980).

[36] H. S. Park, J. S. Baskin, and A. H. Zewail, 4D Lorentz Electron Microscopy Imaging: Magnetic Domain Wall Nucleation, Reversal, and Wave Velocity, Nano Lett. 10, 3796 (2010).

[37] K. B. Schliep, P. Quarterman, J.-P. Wang, and D. J. Flannigan, Picosecond Fresnel Transmission Electron Microscopy, Appl. Phys. Lett. 110, 222404 (2017).

[38] M. Aeschlimann, C. A. Schmuttenmaer, H. E. Elsayed-Ali, R. J. D. Miller, J. Cao, Y. Gao, and D. A. Mantell, Observation of Surface Enhanced Multiphoton Photoemission from Metal Surfaces in the Short Pulse Limit, J. Chem. Phys. 102, 8606 (1995).

[39] H. Dömer and O. Bostanjoglo, High-Speed Transmission Electron Microscope, Rev. Sci. Instrum. 74, 4369 (2003).

[40] A.H. Zewail, Four-Dimensional Electron Microscopy, Science 328, 187 (2010).

[41] R. J. Dwayne Miller, Femtosecond Crystallography with Ultrabright Electrons and X-rays: Capturing Chemistry in Action, Science 343, 1108 (2014).

[42] G. Berruto, I. Madan, Y. Murooka, G. M. Vanacore, E. Pomarico, J. Rajeswari, R. Lamb, P. Huang, A. J. Kruchkov, Y. Togawa, T. LaGrange, D. McGrouther, H. M. Rønnow, and F. Carbone, Laser-Induced Skyrmion Writing and 
Erasing in an Ultrafast Cryo-Lorentz Transmission Electron Microscope, Phys. Rev. Lett. 120, 117201 (2018).

[43] P. Hommelhoff, Y. Sortais, A. Aghajani-Talesh, and M. A. Kasevich, Field Emission Tip as a Nanometer Source of Free Electron Femtosecond Pulses, Phys. Rev. Lett. 96, 077401 (2006).

[44] C. Ropers, D. R. Solli, C. P. Schulz, C. Lienau, and T. Elsaesser, Localized Multiphoton Emission of Femtosecond Electron Pulses from Metal Nanotips, Phys. Rev. Lett. 98, 043907 (2007).

[45] A. Feist, N. Bach, N. R. da Silva, T. Danz, M. Möller, K. E. Priebe, T. Domröse, J. G. Gatzmann, S. Rost, J. Schauss, S. Strauch, R. Bormann, M. Sivis, S. Schäfer, and C. Ropers, Ultrafast Transmission Electron Microscopy Using a Laser-Driven Field Emitter: Femtosecond Resolution with a High Coherence Electron Beam, Ultramicroscopy 176, 63 (2017).

[46] A. Hubert and R. Schäfer, Magnetic Domains-The Analysis of Magnetic Microstructures (Springer, Heidelberg, 2000).

[47] T. Shinjo, T. Okuno, R. Hassdorf, K. Shigeto, and T. Ono, Magnetic Vortex Core Observation in Circular Dots of Permalloy, Science 289, 930 (2000).

[48] J. Raabe, R. Pulwey, R. Sattler, T. Schweinböck, J. Zweck, and D. Weiss, Magnetization Pattern of Ferromagnetic Nanodisks, J. Appl. Phys. 88, 4437 (2000).

[49] N. A. Usov and S. E. Peschany, Magnetization Curling in a Fine Cylindrical Particle, J. Magn. Magn. Mater. 118, L290 (1993).

[50] M. De Graef, Introduction to Conventional Transmission Electron Microscopy (Cambridge University Press, Cambridge, 2003).

[51] Y. Aharonov and D. Bohm, Significance of Electromagnetic Potentials in the Quantum Theory, Phys. Rev. 115, 485 (1959).

[52] M. De Graef and Y. Zhu, Magnetic Imaging and Its Applications to Materials (Academic Press, New York, 2000).

[53] V. V. Volkov, Y. Zhu, and M. De Graef, A New Symmetrized Solution for Phase Retrieval Using the Transport of Intensity Equation, Micron 33, 411 (2002).

[54] See Supplemental Material at http://link.aps.org/ supplemental/10.1103/PhysRevX.8.031052 for movies of ultrafast demagnetization in a single vortex structure, extended experimental details, information on data acquisition and analysis, details on the implementation of Lorentz image simulation and micromagnetic simulations, and discussion on the dependence of image contrast on spin temperature and eletron-probe pulse duration.

[55] A. Feist, K. E. Echternkamp, J. Schauss, S. V. Yalunin, S. Schäfer, and C. Ropers, Quantum Coherent Optical Phase Modulation in an Ultrafast Transmission Electron Microscope, Nature (London) 521, 200 (2015).
[56] A. Feist, N. R. da Silva, W. Liang, C. Ropers, and S. Schäfer, Nanoscale Diffractive Probing of Strain Dynamics in Ultrafast Transmission Electron Microscopy, Struct. Dyn. 5, 014302 (2018).

[57] E. Beaurepaire, J.-C. Merle, A. Daunois, and J.-Y. Bigot, Ultrafast Spin Dynamics in Ferromagnetic Nickel, Phys. Rev. Lett. 76, 4250 (1996).

[58] S. Mathias, C. La-O-Vorakiat, P. Grychtol, P. Granitzka, E. Turgut, J. M. Shaw, R. Adam, H. T. Nembach, M. E. Siemens, S. Eich, C. M. Schneider, T. J. Silva, M. Aeschlimann, M. M. Murnane, and H. C. Kapteyn, Probing the Timescale of the Exchange Interaction in a Ferromagnetic Alloy, Proc. Natl. Acad. Sci. U.S.A. 109, 4792 (2012).

[59] J. Walowski, G. Müller, M. Djordjevic, M. Münzenberg, M. Kläui, C. A. F. Vaz, and J. A. C. Bland, Energy Equilibration Processes of Electrons, Magnons, and Phonons at the Femtosecond Time Scale, Phys. Rev. Lett. 101, 237401 (2008).

[60] Generally, the magnetic configuration is expected to respond to an increased spin temperature in a complex manner due to changes, for example, in the average dipolar and exchange interactions. For the present case, we performed micromagnetic simulations which suggest that only changes in the saturation magnetization need to be considered on ultrashort time scales (see Supplemental Material [54]).

[61] Charles Kittel, Introduction to Solid State Physics 8th ed. (John Wiley \& Sons, New York, 2004).

[62] T. van Oudheusden, P. L. E. M. Pasmans, S. B. van der Geer, M. J. de Loos, M. J. van der Wiel, and O. J. Luiten, Compression of Subrelativistic Space-Charge-Dominated Electron Bunches for Single-Shot Femtosecond Electron Diffraction, Phys. Rev. Lett. 105, 264801 (2010).

[63] R. P. Chatelain, V. R. Morrison, C. Godbout, and B. J. Siwick, Ultrafast Electron Diffraction with RadioFrequency Compressed Electron Pulses, Appl. Phys. Lett. 101, 081901 (2012).

[64] J. Maxson, D. Cesar, G. Calmasini, A. Ody, P. Musumeci, and D. Alesini, Direct Measurement of Sub-10 fs Relativistic Electron Beams with Ultralow Emittance, Phys. Rev. Lett. 118, 154802 (2017).

[65] C. Kealhofer, W. Schneider, D. Ehberger, A. Ryabov, F. Krausz, and P. Baum, All-Optical Control and Metrology of Electron Pulses, Science 352, 429 (2016).

[66] L. Wimmer, G. Herink, D. R. Solli, S. V. Yalunin, K. E. Echternkamp, and C. Ropers, Terahertz Control of Nanotip Photoemission, Nat. Phys. 10, 432 (2014).

[67] K. E. Priebe, C. Rathje, S. V. Yalunin, T. Hohage, A. Feist, S. Schäfer, and C. Ropers, Attosecond Electron Pulse Trains and Quantum State Reconstruction in Ultrafast Transmission Electron Microscopy, Nat. Photonics 11, 793 (2017).

[68] Y. Morimoto and P. Baum, Attosecond Control of Electron Beams at Dielectric and Absorbing Membranes, Phys. Rev. A 97, 033815 (2018). 